\title{
Assessment of the Influence of Photodiode Size on RSS-Based Visible Light Positioning Precision
}

\author{
Willem Raes \\ KU Leuven \\ ESAT-DRAMCO \\ Ghent, Belgium \\ willem.raes@kuleuven.be
}

\author{
Sander Bastiaens \\ Ghent University/imec \\ WAVES-INTEC \\ Ghent, Belgium \\ sander.bastiaens@UGent.be
}

\author{
David Plets \\ Ghent University/imec \\ WAVES-INTEC \\ Ghent, Belgium \\ david.plets@UGent.be
}

\author{
Nobby Stevens \\ KU Leuven \\ ESAT-DRAMCO \\ Ghent, Belgium \\ nobby.stevens@kuleuven.be
}

\begin{abstract}
This work discusses the influence of a photodiodes effective area size on the precision of received signal strengthbased visible light positioning. It analyzes how two silicon-based photodiodes with different effective area perform as a receiver under varying illumination conditions. The two main findings are that it is not particularly needed to select a photodiode with a large surface area, despite the higher received signal strength, due to a higher noise contribution. On the other hand, the spread on the distance estimation is much smaller than $1 \mathrm{~mm}$ under standard illumination levels for the two photodiodes with a significantly different surface area, so that both photodiodes deliver enough precision.
\end{abstract}

\section{INTRODUCTION}

Visible Light Positioning (VLP) is a very promising technology to deliver indoor location based services [1]. It can simultaneously provide highly accurate localization and illumination in a building. The application domain for this technology is very broad. It ranges from indoor logistics in industry where asset and vehicle tracking or navigation is important, to the retail industry where targeted advertisement and customer heat maps are of interest. Many articles with different approaches to attain a visible light positioning system have already been published [2]. Some solutions use a camera or image sensor to obtain angular information from the emitted modulated light. The Received Signal Strength (RSS) based approach [3] that is envisioned in this work uses a single photodiode as receiver. The use of a photodiode as receiver for a VLP-system is well described in literature [4], however the influence of the diodes effective area on the precision of visible light positioning has not yet been investigated experimentally. In this work two commercially available photodiodes with different effective area are considered and the effect of the photodiode area on the precision of RSS-VLP is investigated. Section II describes the measurement setup and the used hardware, while section III discusses the results of the measurements. The main conclusions can be found in section IV.

\section{System Description}

To estimate the influence of the photodiode area $\left(A_{r}\right)$ on the precision of visible light positioning, the measurement setup as shown in Fig. 1, was constructed. An LED ${ }^{1}$, which is modelled

\footnotetext{
${ }^{1}$ Bridgelux BXRE-C3001-D24
}

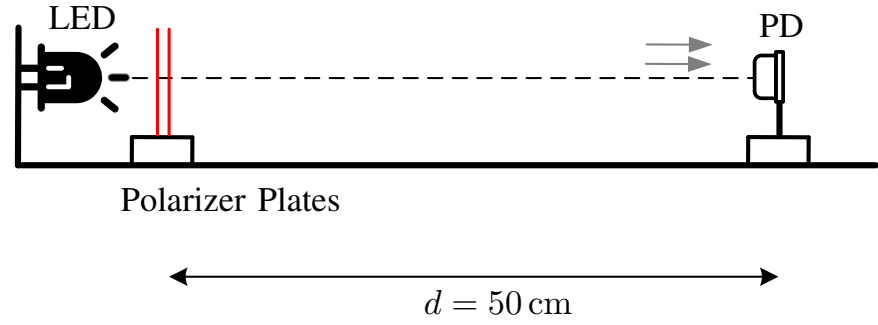

Fig. 1. The experimental setup with the Bridgelux LED to the left of the two polarizer plates. The receiving photodiode (PD) is placed at $50 \mathrm{~cm}$ to the right of the plates. Two commercially available photodiodes with different surface area were used in this setup in order to compare the precision.

as a lambertian radiator of order $m=1$, is configured to emit a power switched waveform. The commercially available receiving photodiode (PD) is placed on a distance $d=50 \mathrm{~cm}$ from the transmitting LED. To modify the transmitted optical power, two linear polarizer plates ${ }^{2}$ were mounted just in front of the LED. By changing the rotation angle between the two polarizer plates, a smooth modification of the incident power at the receiver side is realized. The electrical signal at the photodiode is given by (1) [5].

$$
r(t)=\alpha R_{p} s(t)+\beta+n(t)
$$

Here $R_{p}$ is the PD responsivity, $\beta$ is the dc-contribution of ambient light sources, $\alpha$ is the channel attenuation defined by (2) [6], in which $m=1$ was taken into account. In our optical bench of Fig. 1, the distance $d$ remained unchanged and equal to $50 \mathrm{~cm} . s(t)$ is the transmitted optical power signal after attenuation by the polarizer plates and is described by (3), corresponding with a power switched wave with duty cycle $\delta=0.5$ and periodicity $T_{0}=f_{0}^{-1}$. The optical power $P_{o}$ is easily modified by changing the relative angle between the two linear polarization plates.

$$
\begin{gathered}
\alpha=\frac{A_{r}}{\pi d^{2}} \\
s(t)=\sum_{k=-\infty}^{\infty} P_{o} \operatorname{rect}\left(\frac{t}{T_{0}}-\frac{1}{2}-2 k\right)
\end{gathered}
$$

\footnotetext{
${ }^{2}$ Edmund Optics M43 x 0.75 Mounted Linear Glass Polarizing Filter
} 
The noise at the detector $n(t)$ is the sum of shot noise and Johnson noise. Shot noise is a Poisson process, which can be approximated by a Gaussian distribution for large numbers [7] (in this case a large photon count). Thus one can state that $n(t)$ is a sample of Gaussian noise with expectation value $\mathbb{E}\{n(t)\}=0$ and variance $\sigma^{2}$.

For the conducted measurements, the illumination levels $\left(E_{v}\right)$ were chosen to vary between $100 \mathrm{~lx}$ and $400 \mathrm{~lx}$, with a measurement step of $50 \mathrm{~lx}$. Standards for lighting requirements impose that there is a minimum of $100 \mathrm{~lx}$ in company transportation zones like large warehouses and hallways [8]. Asset tracking and logistics are important use cases for visible light positioning, therefore the experiments described in this work are in correspondence with these illumination levels. To asses the effect of the PD area $A_{r}$ on the precision of visible light based localization, the spread $\sigma_{\hat{d}}$ on the distance estimation $\hat{d}$ is used as a metric. To obtain the power contribution of the LED in the received signal $r(t)$, the Fast Fourier Transformbased demodulation (FFT) was selected [9]. Herefore, $r(t)$ is sampled with a data acquisition module ${ }^{3}$ at a sampling frequency $f_{s}$ of $128 \mathrm{kHz}$ (taking into account a frequency $f_{0}$ of $500 \mathrm{~Hz}, 256$ samples are thus obtained in one period). The average received signal $P_{s}$ is assessed by evaluating the expectation value of the first harmonic component of the modulus of the FFT of $r(t)$ as defined in (4). Averaging over 1000 periods leads to the expectation value.

$r=\bmod \left\{f_{0} \int_{T_{0}} r(t) \exp \left(-j \frac{2 \pi t}{T_{0}}\right) \mathrm{d} t\right\}=\frac{A_{r} R_{p} P_{o}}{\pi^{2} d^{2}}+n$

Since $\mathbb{E}\{n\}=0,(5)$ is valid.

$$
P_{s}=\mathbb{E}\{r\}=\frac{A_{r} R_{p} P_{o}}{\pi^{2} d^{2}}
$$

When a single measurement is executed and the received signal is evaluated, it is to be considered mathematically as taking a sample value of a Gaussian distribution around $P_{s}$ with the variance equal to the noise power. Taken into account the knowledge of $P_{s}$ as the mean of the Gaussian distribution, the estimation of the received signal $\hat{r}$ is described by (6)

$$
\hat{r}=\left(\frac{d}{\hat{d}}\right)^{2} P_{s}
$$

so that an estimation of the distance $\hat{d}$ is easily found (7) for each sample of the received power.

$$
\hat{d}=d \sqrt{\frac{P_{s}}{\hat{r}}}
$$

Using this method to obtain a distance estimation for every acquired signal period allows us to assess the precision on $\hat{d}$ and thus the spread on the measurements for both receiver modules.

$$
\sigma_{\hat{d}}^{2}=\operatorname{Var}\{\hat{d}\}
$$

\footnotetext{
${ }^{3}$ USB6212 National Instruments
}

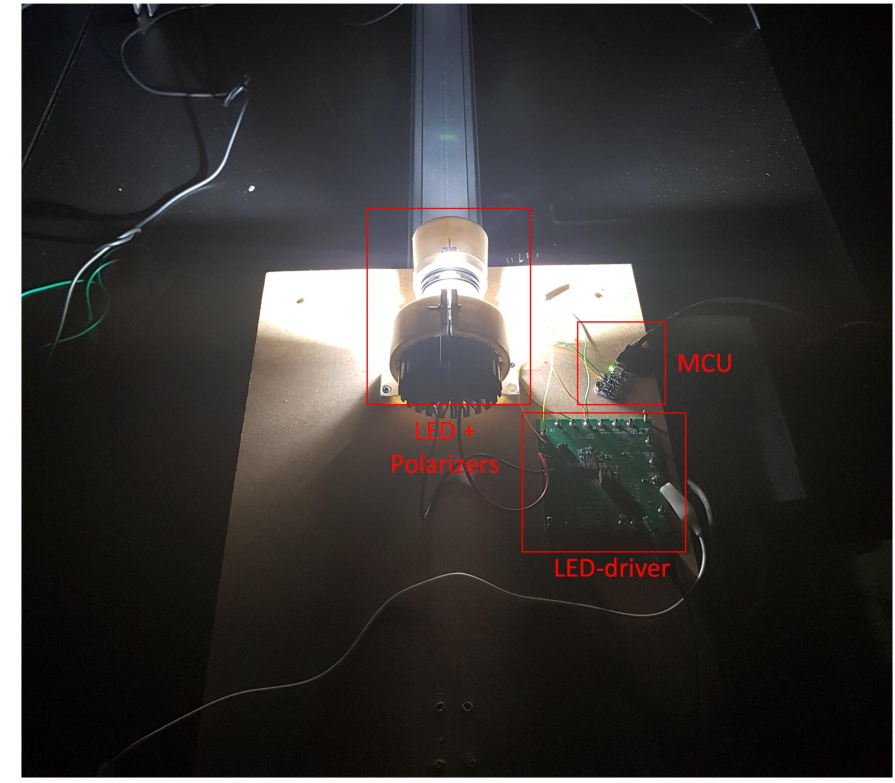

Fig. 2. The transmitter side of the measurement setup with the mounted LED, LED-driver and microcontroller unit for signal generation. The polarizer plates are mounted just in front of the LED.

\section{Results AND Discussion}

The measurements were conducted with the two commercially available modules from manufacturer Thorlabs, the PDA36A $2^{4}$ and the PDA100A ${ }^{5}$. The modules have a built-in transimpedance amplifier (TIA) and they were chosen because of their excellent traceability in terms of noise properties and responsivity. The receiver diodes are configured in photoconductive mode, hence with a reverse bias applied. Their effective area is $13 \mathrm{~mm}^{2}$ (PDA36A2) and $100 \mathrm{~mm}^{2}$ (PDA100A). To maximize the dynamic range of the analog-to-digital converter (ADC), the transimpedance gain of the PDA36A2 was set to $30 \mathrm{~dB}$ and that of the PDA100A to $20 \mathrm{~dB}$. Fig. (2) shows the transmitter portion of the measurement setup and clarifies how the LED and polarizer plates were mounted.

As can be observed from Fig. 3 the spread on the distance estimation $\sigma_{\hat{d}}$ globally declines with increasing illumination level for both receiver modules. It is also found that the smaller PD (PDA36A2) has a higher precision over the entire measurement interval. However, the order of magnitude of $\sigma_{\hat{d}}$ and thus the spread on the distance estimation is significantly lower than $1 \mathrm{~mm}$, leading to the result that actually both photodiodes qualify to be used for accurate indoor positioning under normal illumination levels. The normalized histograms and fitted Gaussian distributions in Fig. 4 and Fig. 5 show the spread on the distance estimation $\hat{d}$ at illumination level $E_{v}=400 \mathrm{~lx}$ for respectively, the PDA100A and PDA36A2 receiver modules. The histograms have been normalized so that the integral over the measurement set sums to one in order to facilitate the comparison with a probability distribution.

\footnotetext{
${ }^{4}$ https://www.thorlabs.com/thorproduct.cfm?partnumber=PDA36A2 ${ }^{5}$ https://www.thorlabs.com/thorproduct.cfm?partnumber=PDA100A
} 


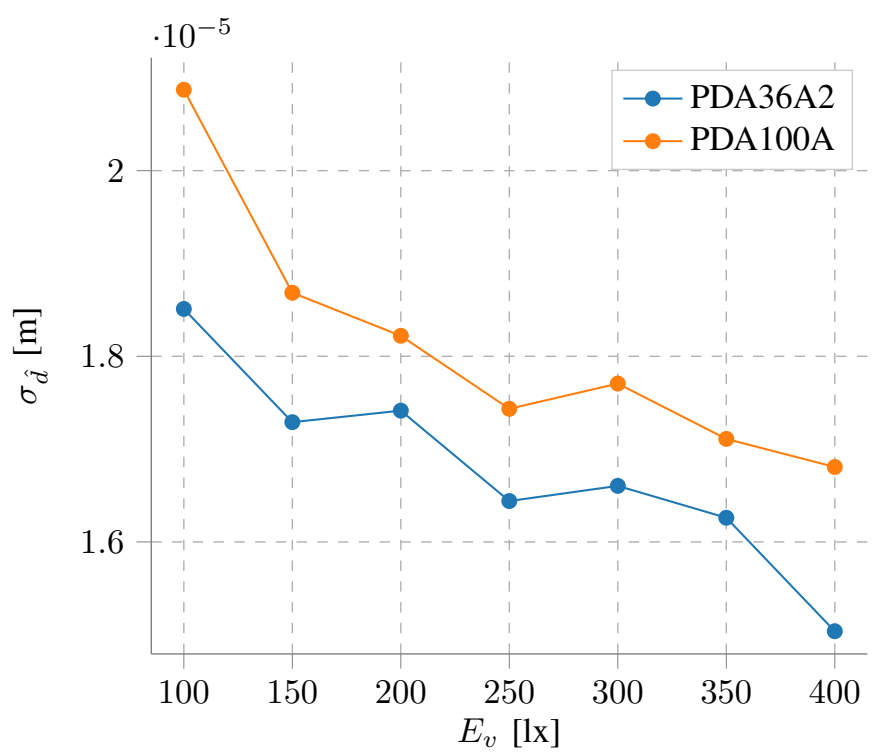

Fig. 3. The standard deviation $\sigma_{\hat{d}}$ as a function of the illuminance at the receiver $E_{v}$ for the two photodiodes under study.

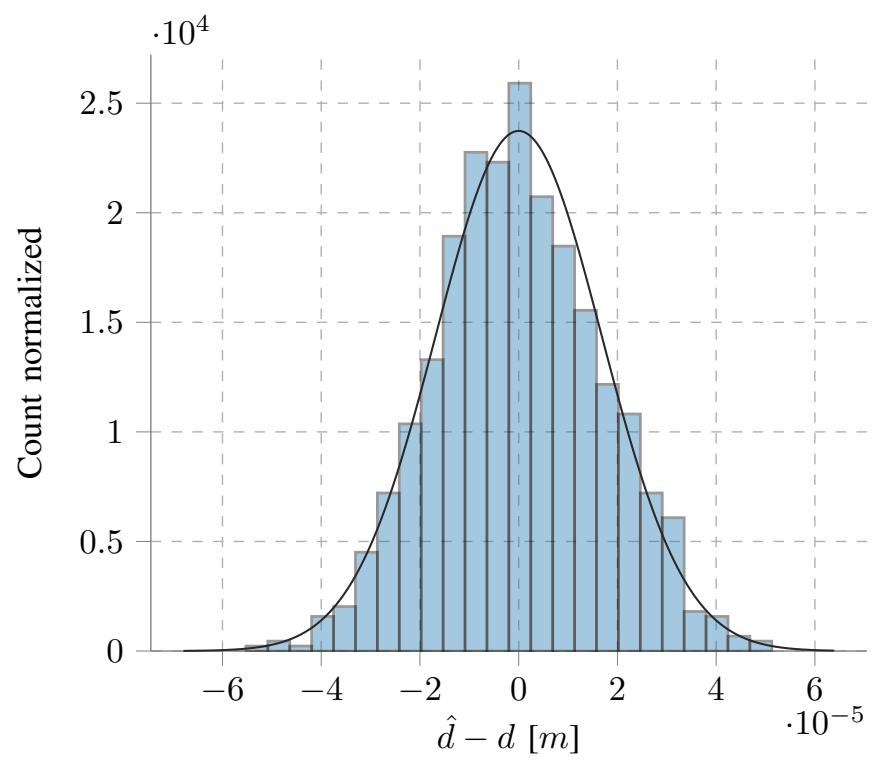

Fig. 4. Normalized histogram of the distance estimation $\hat{d}-d$ with the PDA100A receiver module at an illumination level of 400 lux.

\section{CONCLUSiON}

In this work the effect of the area size of a photodiode on the precision of RSS-based Visible Light Positioning has been experimentally investigated. The measurements focus on illumination levels that are standard for transportation zones in company warehouses where logistics are important. It is found that, despite a significant difference in surface area (almost a factor eight), both photodiodes deliver results where the standard deviation on the distance estimation is significantly below $1 \mathrm{~mm}$. As a main conclusion, one can thus state that the impact of the surface area of both commercially available photodiodes on the precision is not a relevant selection criterion.

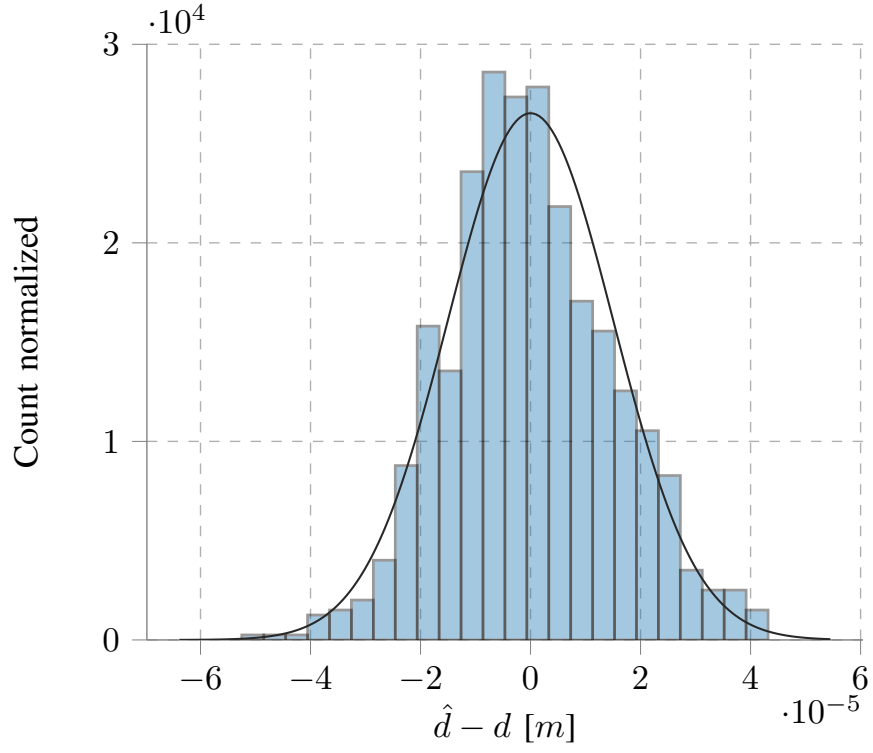

Fig. 5. Normalized histogram of the distance estimation $\hat{d}$ with the PDA36A2 receiver module at an illumination level of 400 lux.

\section{ACKNOWLEDGMENT}

This work was executed within LEDsTrack, a research project bringing together academic researchers and industry partners. The LEDsTrack project was co-financed by imec and received project support from Flanders Innovation \& Entrepreneurship.

\section{REFERENCES}

[1] C. Wang, L. Wang, X. Chi, S. Liu, W. Shi and J. Deng, The research of indoor positioning based on visible light communication, in China Communications, vol. 12, no. 8, pp. 85-92, August 2015.

[2] J. Luo, L. Fan and H. Li, Indoor Positioning Systems Based on Visible Light Communication: State of the Art, in IEEE Communications Surveys \& Tutorials, vol. 19, no. 4, pp. 2871-2893, Fourthquarter 2017.

[3] X. Zhang, J. Duan, Y. Fu, A. Shi, "Theoretical Accuracy Analysis of Indoor Visible Light Communication Positioning System Based on Received Signal Strength Indicator," Journal of Lightwave Technology, Vol. 32, No. 21, Nov 2014, pp. 4180-4186.

[4] Do T.-H., Yoo, M. An in-Depth Survey of Visible Light Communication Based Positioning Systems, in Sensors 2016, vol. 16, no. 5.

[5] M. F. Keskin and S. Gezici, Comparative Theoretical Analysis of Distance Estimation in Visible Light Positioning Systems, in Journal of Lightwave Technology, vol. 34, no. 3, pp. 854-865, Feb.1, 12016.

[6] T. Komine and M. Nakagawa, Fundamental analysis for visible-light communication system using LED lights, in IEEE Transactions on Consumer Electronics, vol. 50, no. 1, pp. 100-107, Feb 2004.

[7] J. M. Kahn and J. R. Barry, Wireless infrared communications, in Proceedings of the IEEE, vol. 85, no. 2, pp. 265-298, Feb 1997.

[8] Comité Européen de Normalisation (CEN). 2002. CEN Standard EN 12464-1: Light and Lighting Lighting of Work Places Part 1: Indoor Work Places, Brussels: CEN

[9] S. De Lausnay, L. De Strycker, J. P. Goemaere, N. Stevens and B. Nauwelaers, A Visible Light Positioning system using Frequency Division Multiple Access with Square Waves, 2015 9th International Conference on Signal Processing and Communication Systems (ICSPCS), Cairns, QLD, 2015, pp. 1-7.

[10] A. Kupper, Location-based Services: Fundamentals and Operation, isbn 0470092319, John Wiley \& Sons, 2005. 